\title{
RÉALISATION D'UNE INSTRUMENTATION DESTINÉE À L'ÉTUDE DE L'OTO-ÉMISSION ACOUSTIQUE
}

\author{
F. LE FRIOUS et B. ARAGON \\ Laboratoire d'Acoustique, de Métrologie et d'Instrumentation, 38 Rue des 36 Ponts, \\ F-31400 Toulouse, France
}

\begin{abstract}
RESUME : $\quad$ Nous nous interessons à la partie physique de l'oto-émission. Dans un premier temps, nous avons étudié les sondes de détection. Pour cela nous avons spécialement réalisé une cavité d'étalonnage. Les principales mesures ont été les suivantes : courbes de réponses en fréquences, étude de la linéarité des transducteurs, influence du volume sur lequel débouche la sonde, influence de la longueur des capillaires qui prolongent les transducteurs. Ensuite, nous avons réalisé un système d'acquisition et de traitement de l'oto-émission. Nous présentons ici les différentes parties de ce système ainsi que quelques signaux recueillis.
\end{abstract}

\begin{abstract}
We are interesting in the physical part of the acoustic otoemissions. The otoemission probes have first been studied. For this to be done, a calibrating cavity has been specialy made. The measurements made are : plots of the frequency characteristics of the emitters and the receivers, study of the influence of the volume into which the probe emerges, study of the influence of the length of the capillary prolonging each transducer. Then an otoemission detection and processing device has been implemented. The different parts of this system are described and some recorded signals are shown in this paper.
\end{abstract}

\section{I - LES OTO-EMISSIONS PROVOQUEES}

En 1978, KEMP recueille un son provenant de l'oreille après l'avoir stimulée avec un son de très courte durée [1]. Dans les années 80, ce phénomène est enregistré par plusieurs chercheurs : ZWICKER (83), ZUREK (85), GRANDORI (86), PROBST , PUJOL ...

Ce phénomène, appelé oto-émission acoustique provoquée, présente principalement les caractéristiques suivantes :

- Le temps de latence est variable selon les individus et se situe entre 3 et $10 \mathrm{~ms}$.

- Le signal émis varie linéairement avec l'amplitude de stimulation et présente une saturation lorsque la stimulation est supérieure au seuil d'audition de celle-ci de 30 à $40 \mathrm{~dB}$.

- L'allure du signal et donc sa signature fréquentielle sont propres à chaque individu et sont constants dans le temps chez un même individu, sauf en cas de perte d'audition et particulièrement d'atteinte cochléaire. Les fréquences caractéristiques de l'oto-émission se situent entre $800 \mathrm{et} 4000 \mathrm{~Hz}$.

- L'apparition de l'oto-émission aurait lieu pour des niveaux sonores de stimulation inférieurs au seuil de perception de l'indivu.

L'origine de l'oto-émission est expliquée par les découvertes récentes en physiologie cochléaire [2]:

Nous savons maintenant que seules les cellules ciliées externes sont solidaires de la membrane tectoriale. Leurs cils sont actionnés proportionnellement au déplacement de la membrane basilaire, tandis que les cils des cellules ciliées internes sont actionnés par les déplacements liquidiens et les forces de viscosité, leur déplacement étant proportionnel à la vitesse de déplacement de la membrane basilaire [3].

Une autre découverte importante a été la mise en évidence de protéines contractiles dans les cellules ciliées externes : actine et myosine, et dans les racines des cils : tropomyosine et calmoduline [4]. Des stimulations des cellules ciliées externes provoquent des variations de volume et des raccourcissements significatifs de ces cellules [5]. 
Aussi donne-t-on une nouvelle explication du mécanisme de transduction cochléaire. Ce mécanisme permet d'augmenter la sélectivité fréquentielle aux faibles niveaux sonores et cela permet de comprendre la meilleure sélectivité en fréquence que ce qu'avait prévu BEKESY. Ce phénomène actif endocochléaire, qu'est la contraction des cellules ciliées externes, semble actuellement être directement impliqué dans la génèse de l'oto-émission acoustique.

\section{II - ETUDE DES SONDES DE DETECTION}

\section{II.1 - Description des sondes}

Pour recueillir une oto-émission, il faut stimuler l'oreille avec un son bref à l'aide d'un émetteur, et à l'aide d'un microphone on capte le signal d'excitation suivi du signal de l'oto-émission. Ces deux transducteurs doivent être de petite taille pour être placés tous les deux dans le conduit auditif. Ces transducteurs sont les mêmes que ceux utilisés dans les prothèses auditives, mais l'assemblage et l'environnement sont propres aux sondes d'oto-émissions.

L'émetteur est de type électromagnétique et le récepteur est un transducteur électrostatique à électret. Ils sont placés côte-à-côte dans un boitier ou dans un moulage selon le constructeur.

Un bouchon d'oreille en caoutchouc, placé à l'extrémité de la sonde, assure l'étanchêité dans le conduit auditif.

\section{II.2 - Dispositif d'étalonnage}

Etalonner une sonde d'oto-émissions revient à étalonner un microphone et un émetteur. Il s'agit de réaliser un étalonnage en pression. Cela implique que la pression soit uniforme dans la cavité d'étalonnage pour les fréquences d'utilisation $(800-4000 \mathrm{~Hz})$, pour éviter l'apparition de mouvement ondulatoire et de phénomène stationnaire. De plus la cavité doit être adaptée à notre problème :

- Le volume doit avoir les dimensions du conduit auditif fermé par un bouchon d'oreille.

- Le microphone de référence ne doit pas être démesurément grand devant la surface du tympan $\left(70 \mathrm{~mm}^{2}\right)$, mais il doit avoir une bonne sensibilité.

Pour réaliser le dispositif d'étalonnage, nous nous sommes inspirés d'une norme (IEC 29C42) décrivant une cavité simulant une oreille occluse. Nous avons gardé les dimensions internes de la cavité. Pour le microphone de référence nous avons choisi un microphone Brüel \& Kjær 1/2 pouce, qui nous a semble être un bon compromis entre une bonne sensibilité et une membrane de surface s'approchant de celle du tympan.

Le microphone de référence permet de connaître le niveau de pression sonore dans la cavité, qui est soit fourni par l'émetteur lors de l'étude de l'émission, soit reçu par le récepteur lors de l'étude de la réception.

Une source sonore, reliée à la cavité par un capillaire métallique, permet de fournir un niveau de pression sonore dans la cavité, lors de l'étude du récepteur seul.

Pour tracer les caractéristiques en fréquences des émetteurs et des récepteurs, nous travaillons avec un analyseur de signaux bicanal. Ce type d'analyseur permet donc de calculer des spectres mais aussi des fonctions de transfert, des fonctions de cohérence, ... De plus nous pouvons moyenner les signaux temporels avant de les analyser.

\section{II.3 - Courbes de réponses en fréanences}

Nous avons étudié plusieurs sondes qui ont les caractéristiques morphologiques suivantes :

Les sondes $n^{\circ} 1$ et $n^{\circ} 2$ ont un boitier de sonde d'impédancemétrie. Le récepteur de la sonde $n^{\circ} 2$ est prolongé d'un capillaire métallique jusqu'à l'extrémité de la sonde.

Les sondes $n^{\circ} 3, n^{\circ} 6$ et $n^{\circ} 7$ sont en forme de cone et s'adapte bien à la forme du conduit auditif. Les deux transducteurs sont prolongés de capillaires et sont cellés dans le cone.

Les différents montages sont directement responsables de l'allure des courbes de réponses en fréquences présentées sur la figure 1 . Après cette constatation nous avons étudié plus particulièrement l'influence de la longueur du capillaire qui prolonge le transducteur. Nous concluons que la longueur doit être la plus courte possible pour rejeter la fréquence de résonance vers les hautes fréquences [6].

De même, nous avons étudié l'influence du volume sur lequel débouche la sonde. Nous constatons que ce volume doit être le plus faible possible pour rejeter la fréquence d'anti-résonance vers les hautes fréquences [6]. 

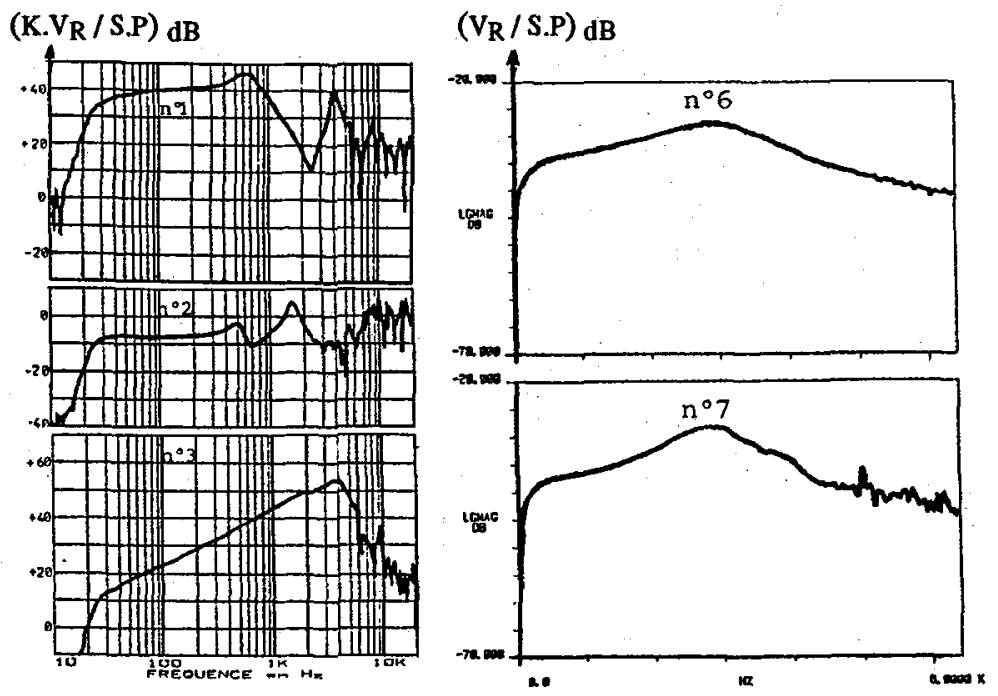

Figure 1: Courbes de réponses en fréquences de quelques récepteurs.

\section{III - DISPOSITIF D'ACQUISITION DE L'OTO-EMISSION}

Une fois que nous connaissions bien les sondes de détection, nous désirions mettre au point notre propre instrumentation pour l'acquisition de l'oto-émission. Notre but est de monter une instrumentation de recherche, où la plupart des paramètres d'acquisition sont modifiables. De plus, nous proposons de quantifier le signal de l'oto-émission en connaissant les caractéristiques de toutes les parties du dispositif.

Le dispositif réalisé est basé sur l'utilisation d'une carte d'acquisition et de traitement numérique du signal. Cette carte est développée autour d'un processeur ADSP 2100. Elle est implantée dans un micro-ordinateur de type PC AT. Pour la sortie de la carte, c'est-à-dire pour générer l'excitation de l'émetteur, nous utilisons un convertisseur 8 bits $( \pm 1 \mathrm{~V})$. Et pour l'entrée, nous utilisons un convertisseur 12 bits $( \pm 2,5 \mathrm{~V})$.

Mise à part cette carte d'acquisition, tout le dispositif a été conçu et réalisé au LAMI.

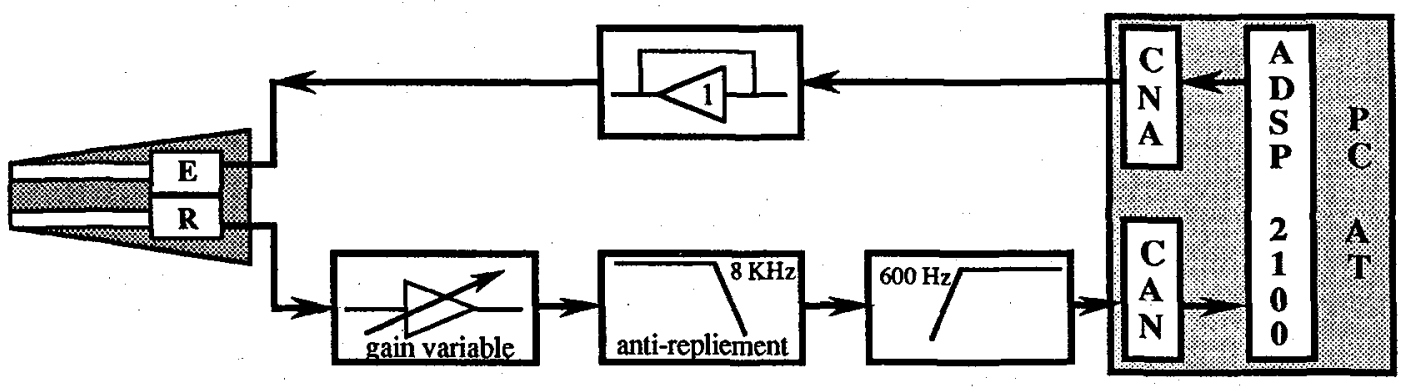

Figure 2 : Schéma synoptique du dispostif d'acquisition.

- Pour la partie acoustique, la sonde est réalisée avec deux transducteurs Knowles. Ils sont prolongés de capillaires souples jusqu'à l'extrémité de la sonde, et sont cellés avec un polymère. La sonde est de forme conique. Il n'y a donc pas de volume résiduel à l'intérieur de la sonde et celle-ci a une forme adaptée à celle du conduit auditif. Nous avons étudié notre sonde dans notre cavité d'étalonnage : sensibilité du récepteur, efficacité de l'émetteur, linéarité et fonction de transfert de chaque transducteur.

- La partie électronique consiste, côté réception, en un amplificateur de gain variable (40 à $120 \mathrm{~dB}$ ), suivi d'un filtre anti-repliement à $8 \mathrm{KHz}$ et d'un filtre passe-haut à $600 \mathrm{~Hz}$. Ce dernier filtre est destiné à éliminer les bruits basses fréquences de l'organisme et les bruits des machines environnantes. 
Côté émission, il s'agit simplement d'un amplificateur suiveur pour assurer l'adaptation d'impédance entre le module de sortie de la carte d'acquisition et l'émetteur. Pour cette partie, nous pouvons donner également les caractéristiques techniques : fonction de transfert, bruit.

- Pour la partie informatique, l'acquisition et le traitement du signal sont programmés en assembleur DSP 2100. L'interface entre le manipulateur et le système d'acquisition, c'est-à-dire le choix des paramètres d'acquisition et la visualisation graphique des signaux temporels et fréquentiels, est programmée en Turbo Pascal. Le signal d'excitation de l'émetteur est une impulsion rectangulaire variable en durée et en amplitude. La tension délivrée par le récepteur, après avoir été amplifiée et filtrée, peut être enregistrée après un délai. Ce délai entre la fin de la stimulation et l'acquisition est choisi par l'opérateur. Pour extraire l'oto-émission du bruit d'origine électronique ou/et acoustique, il est nécessaire de moyenner les signaux temporels ; cela est possible car l'oto-émission est synchronisée avec la stimulation de l'oreille, même si le temps de latence varie d'un individu à l'autre. Le nombre de moyennes à effectuer est également choisi par l'opérateur. Enfin la visualisation graphique du signal est faite après un nombre régulier de moyennes, qui peut être fixé, ce qui permet de suivre l'évolution du signal au cours de la moyenne. Le cas où il y aurait saturation du convertisseur d'entrée a été géré par un rejet total de l'acquisition. Celle-ci n'est donc pas comptée dans le nombre de moyennes mais dans le nombre de rejets. Si le nombre de rejets est trop important, il est nécessaire d'adapter le gain de l'amplificateur pour ne pas trop ralonger la durée de l'expérimentation, voire de la rendre possible. La moyenne étant terminée, un programme de transformée de Fourier rapide permet la visualisation du spectre du signal recueilli.

Nous présentons ici quelques signaux recueillis sur des individus normaux-entendants. Nous sommes actuellement dans la phase d'analyse des résultats et au moment de cette publication, nous ne sommes pas en mesure de donner davantage de précision sur les signaux obtenus. Les signaux présentés ici ont été recueillis avec le dispositif décrit ci-dessus avec l'amplificateur réglé sur $80 \mathrm{~dB}$.

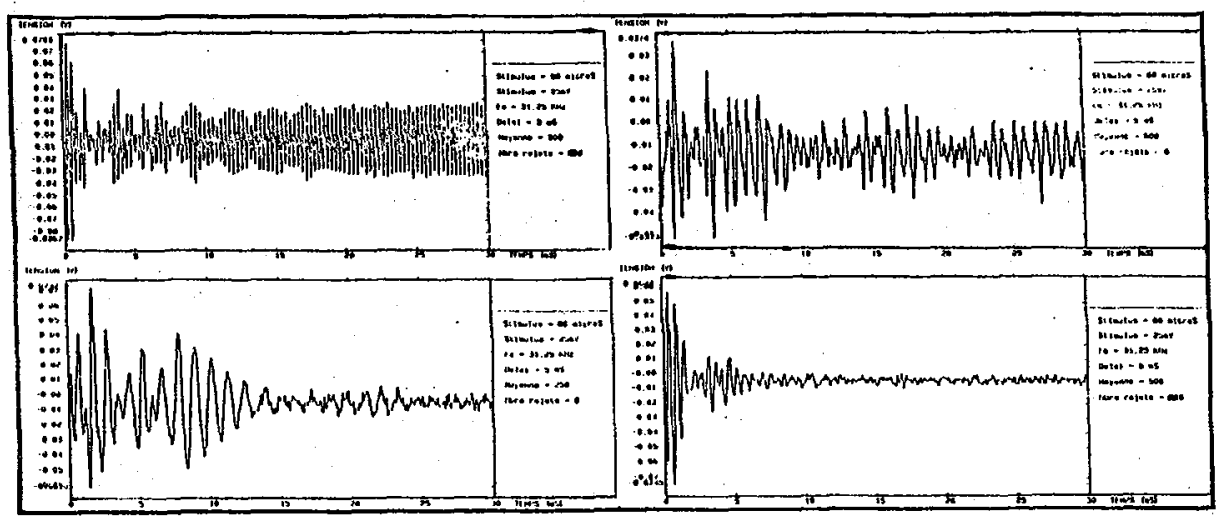

Figure 3 : Exemples de signaux recueillis.

\section{Remerciements_pour_leur participation_à ce trayail :}

Mr Pierre TELLA pour la réalisation mécanique du dispositif d'étalonnage.

Mr André MAYET pour la réalisation électronique du dispositif d'acquisition.

\section{Références_bibliogranhiques :}

[1] D.T. KEMP - "Stimulated acoustic emissions from within the human auditory system." J.A.S.A. 78,64,1386-1391 .

[2] R. PUJOL "Anatomie fonctionnelle de la cochlée" - Physiologie de la cochlée Edition INSERM/SFA - chap.1, 5-26.

[3] BONFILS , PUJOL , REBILLARD, UZIEL - "Données récentes sur la physiologie cochléaire." - Revue de la littérature - IPSEN 1988.

[4] FLOCK - "Hearing" - Physiological bases and Psychophysics 83,2,5 .

[5] BROWNELL - "Mechanisms of hearing" - Webster \& Aitkin Ed. 1983.

[6] F. LE FRIOUS , B. ARAGON - "Caractérisation de sondes d'oto-émissions" Premier congrès français d'acoustique 1990 - vol. 2 , 935-938. 